\title{
Harninkontinenz bei COPD
}

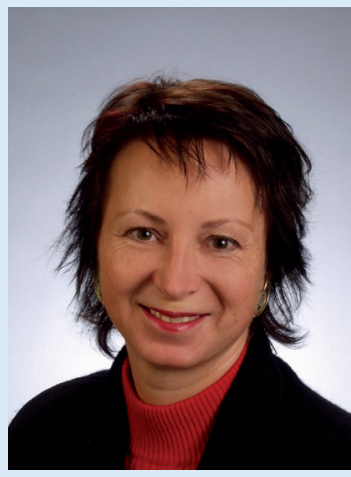

\section{Barbara Köhler}

Dozentin an der Zürcher Hochschule für angewandte Wissenschaften (ZHAW), Winterthur, Schweiz

Harninkontinenz bei COPD

\section{Einleitung}

Ergebnisse der Umfrage der Patientenorganisation Lungenemphysem-COPD Deutschland und des Westdeutschen Lungenzentrums, Ruhrlandklinik, Universitätsklinikum Essen

Bei vielen Menschen mit COPD treten Begleiterkrankungen wie z.B. Osteoporose, Depressionen und Herz-Kreislauferkrankungen auf. In der täglichen Praxis fallen uns immer wieder Patientinnen und Patienten mit chronischen Lungenerkrankungen auf, die zusätzlich unter Harninkontinenz leiden. Harninkontinenz als mögliche Begleiterscheinung chronischer Lungenerkrankungen wurde bislang in der Wissenschaft kaum beachtet.

\section{Was haben wir untersucht?}

Wir sind in unserer Umfrage in Zusammenarbeit mit der "Patientenorganisation Lungenemphysem-COPD Deutschland" und dem „Westdeutschen Lungenzentrum, Ruhrlandklinik, Universitätsklinikum Essen" der Frage nachgegangen, ob Menschen mit COPD möglicherweise häufiger und stärker von Harninkontinenz betroffen sind. Zu diesem Zweck wurden Patientinnen und Patienten der Ruhrlandklinik mit COPD und zum Vergleich eine weitere Anzahl Patientinnen und Patienten mit Tumorentfernung bei Lungenkrebs zu Symptomen von Harninkontinenz befragt. Parallel dazu lief eine Online-Befragung über die Patientenorganisation Lungenemphysem-COPD Deutschland.

Zum Zeitpunkt der Erstellung dieses Textes für die Kongresszeitung 2013 war die Online-Befragung noch nicht abgeschlossen. Definitive Ergebnisse werden auf dem Symposium-Lunge 2013 präsentiert werden. Erste Analysen unserer Umfrage zeigen jedoch, dass Harninkontinenz bei Menschen mit COPD deutlich häufiger und bereits im mittleren Alter auftritt, im Vergleich zur Kontrollgruppe unserer Studie oder zu bekannten Erhebungen in der deutschen Bevölkerung.

\section{Was genau ist Harninkontinenz und warum tritt} sie auf?

Harninkontinenz wird von der Internationalen Kontinenzgesellschaft (ICS) als ,jeglicher unfreiwilliger Verlust von Harn" bezeichnet. Etwa 8 Millionen Menschen in Deutschland leiden unter Harninkontinenz. Die häufigste Form von Harninkontinenz bei Frauen ist die Belastungsinkontinenz (ehemals Stressinkontinenz), die durch Druckerhöhung im Bauchraum durch Lachen, Husten, Niesen, Gehen oder Hüpfen auftritt. Bei Männern mit Harninkontinenz ist die Dranginkontinenz am häufigsten anzutreffen, bei der ein zu starker Harndrang im Verhältnis zur entleerten Harnmenge auftritt und bei der auf dem Weg zur Toilette häufig Harn verloren wird. Auch eine Mischform beider Erkrankungen ist möglich.
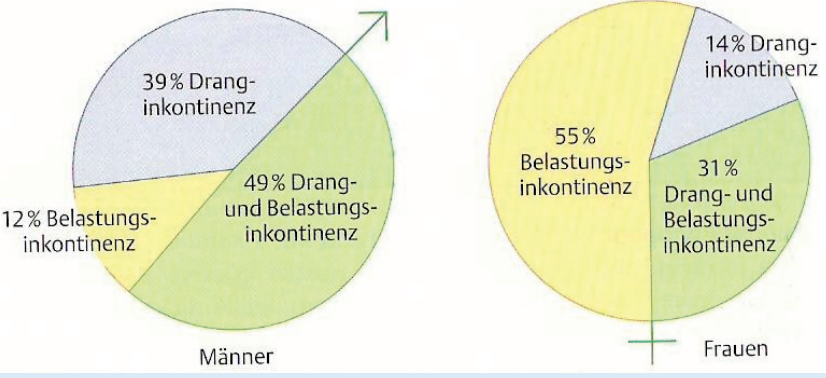

aus Sökeland et al 2007

Die Hauptursache für die Entwicklung einer Belastungsinkontinenz ist eine chronische Erhöhung des Druckes im Bauchraum, wie zum Beispiel beim Husten. Frauen sind wegen des breiteren Beckens und wegen der Folgen von Schwangerschaft und / oder Wechseljahren deutlich stärker gefährdet als Männer. In der deutschen Bevölkerung leiden etwa 55\% der inkontinenten Frauen und etwa $12 \%$ der inkontinenten Männer unter Belastungsinkontinenz.

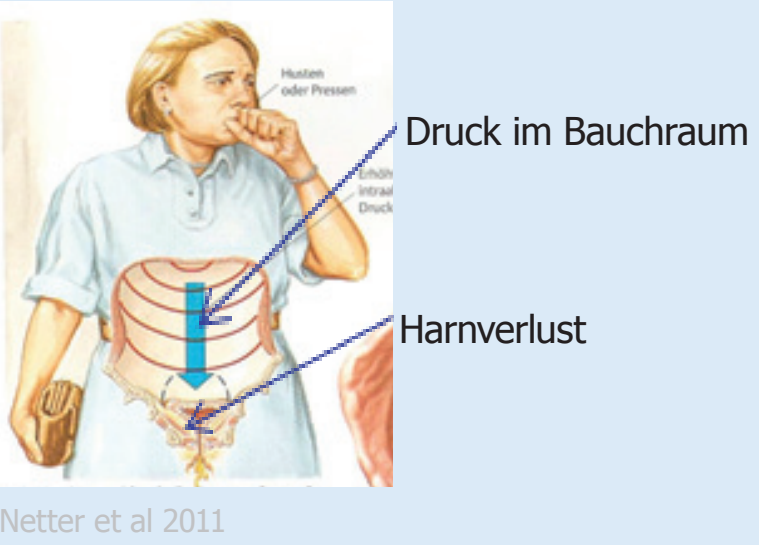

Hoher Druck im Bauchraum braucht eine kräftige Schliessmuskulatur der Blase und eine aktive Beckenbodenmuskulatur, um die Kontinenz zu gewährleisten. 
Scham und sozialer Rückzug sind bei Menschen, die unter Harninkontinenz leiden, häufig eine schwere Belastung. Menschen, die von Harninkontinenz betroffen sind, trinken häufig zu wenig - 2 Liter täglich sollte jeder Mensch trinken. In der Folge verliert die Blase die Fähigkeit, das normale Harnvolumen aufnehmen zu können, und wird zu empfindlich, d.h. es wird zusätzlich eine Dranginkontinenz entwickelt.

Spezialisierte Physiotherapie bietet effektive und kostengünstige Prävention und Rehabilitation. Beratung zum Trink- und Entleerungsverhalten, sowie gezieltes Training der Beckenbodenmuskulatur ist effektiv und der wissenschaftliche Nachweis wurde bereits erbracht.

In unserer Umfrage ist die Tendenz ersichtlich, dass der grösste Teil der Teilnehmenden, die von Harninkontinenz betroffen sind, deswegen noch nie beim Arzt/ bei einer Ärztin war. Auch war Beckenbodentraining zur Linderung der Inkontinenzbeschwerden bei den Befragten kaum bekannt.

\section{Bin ich betroffen?}

Falls Sie eine der folgenden Fragen mit ,ja" beantworten, empfehlen wir eine urologische oder gynäkologische Abklärung beim Facharzt:

\section{Checkliste Harninkontinenz}

Lassen Sie tagsüber mehr als 7 Mal Wasser?

Lassen Sie nachts mehr als 1-2 Mal Wasser?

Verlieren Sie Harn während Sie schlafen?

Ist Ihr Harndrang so stark, dass Sie sofort zur Toilette müssen?

Verlieren Sie Harn, bevor Sie die Toilette erreichen?

Verlieren Sie Harn beim Lachen, Husten, Niesen oder Sport - insbesondere Lungensport?

Ist Ihr Harnstrahl schwach, langsam oder verlängert?

Haben Sie das Gefühl, dass Sie die Blase nicht vollständig entleeren?

Müssen Sie pressen, um Harn zu lassen?

Tragen Sie Vorlagen oder Binden wegen Harnverlust?

Schränken Sie die Trinkmenge ein, um Harnverlust zu vermeiden?

Haben Sie Schmerzen beim Wasserlassen?

Haben Sie häufig Blaseninfektionen?

Beeinträchtigt Harnverlust Ihr tägliche Leben wie Einkaufen, Ausgehen, Besuche u.ä.?

\section{Was kann ich tun?}

Führen Sie ein Trink- und Miktionstagebuch! Finden Sie als erstes heraus, ob Sie ein Problem haben und ob Sie sich beraten lassen sollten. Ein entsprechendes Tagebuch sollten Sie in Ihrer Apotheke erhalten, sicherlich aber im Internet finden können. Führen Sie das Tagebuch am besten an 3 aufeinander folgenden Tagen. Sie benötigen neben dem Tagebuch einen Messbecher mit Milliliter-Angaben.

Wünschenswert ist eine Trinkmenge von etwa 2 Litern pro Tag, 7 bis 8 Toilettengänge tagsüber und nachts höchstens 1 bis 2 . Harnverlust sollte nicht auftreten und bei Drang sollte auch bis zum nächsten Toilettengang abgewartet werden können.

\section{Beckenbodentraining}

Nach der medizinischen Abklärung empfehlen wir Ihnen, täglich selbstständig Beckenbodenübungen durchzuführen. Eine exakte Anleitung kann Ihnen jede spezialisierte Physiotherapeutin geben.

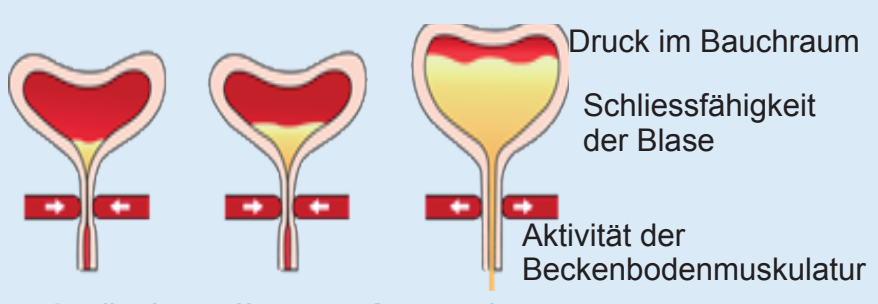

Normaler Weise reicht die Kraft der Schliessmuskulatur und des Beckenbodens aus, um auf Druckerhöhungen im Bauchraum zu reagieren und Harnverlust zu verhindern.

Wenn der Beckenboden und die Schliessmuskulatur versagen, kann es bei Erhöhung des Druckes im Bauchraum zu Harnverlust kommen.

Beckenbodentraining, das durch Physiotherapeutinnen mit einer Zusatzausbildung angeboten wird, kann diese Muskelschwäche verbessern und somit die Symptome der Harninkontinenz lindern oder beseitigen.

Beckenbodentraining ist eine bewusste Anspannung und Entspannung des Beckenbodens. Um diese Muskeln bewusst anspannen zu können, müssen sie wahrgenommen und aktiviert werden können.

Es gibt eine Vielzahl von Übungen, die man auch im Internet suchen kann. Nicht alle dieser Übungen sind für jeden Menschen wirklich sinnvoll. Eine individuelle Schulung durch spezialisierte Physiotherapeutinnen ist deshalb zu empfehlen. 


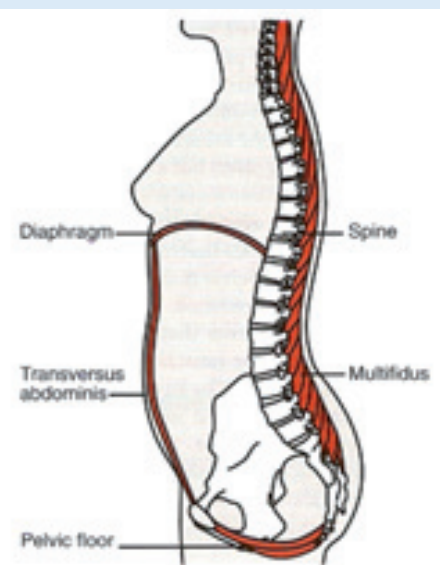

aus Baessler et al 2005

Die Beckenbodenmuskulatur schliesst das Becken nach unten ab und hält die Bauchorgane, insbesondere die Harnblase, in der korrekten Position. Die tiefen Bauchund Rückenmuskeln und das Zwerchfell sollten ausgewogen mit dem Beckenboden zusammen arbeiten.

\section{Startübung}

Im aufrechten Sitz soll der Beckenboden mit seiner Muskulatur gespürt werden. Man kann auf einer Rolle oder einem zusammengerollten Handtuch sitzen.

Bewegen Sie nun langsam das Becken in verschiedene Richtungen und spüren den Druck der Rolle auf Ihrem Beckenboden. Wenn Sie anschliessend die Rolle entfernen und sich wieder hinsetzen, werden Sie den Beckenboden deutlicher spüren. Spannen Sie nun den Beckenboden an, indem Sie sich vorstellen, die Harnröhre und den After zu schliessen, so als möchten Sie Harn oder Darmluft zurückhalten. Halten Sie die Spannung für 1-2 Sekunden und lassen dann bewusst locker. Atmen Sie beim Anspannen aus - beim Einatmen lassen Sie locker. Wiederholen Sie die Übung 10 Mal und führen dies 3 Mal täglich durch.

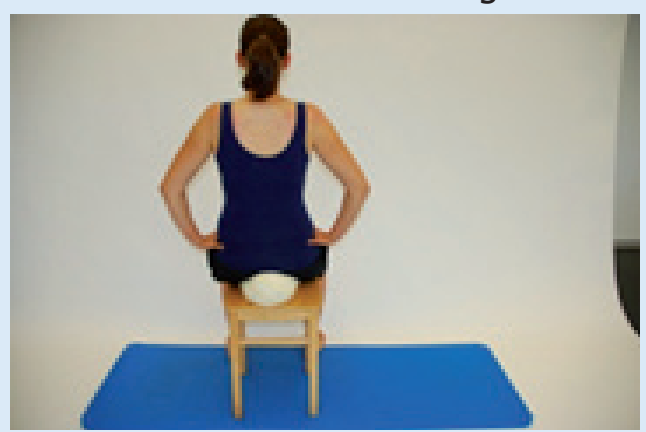

\section{Spezialübung für Patientinnen und Patienten mit COPD}

Patientinnen und Patienten mit obstruktiven Lungenerkrankungen empfehlen wir, die Startübung mit dem Einsatz eines Atemtrainingsgerätes - zum Beispiel Peakflow - oder durch Husten. Der Beckenboden wird vor und während des Ausatemstosses oder Hustens bewusst angespannt. Im Alltag ist diese Strategie äusserst hilfreich, um Harnverlust zu verhindern.
Das Übungsrepertoire kann beliebig erweitert werden. Nach erfolgreich kontrolliertem Einzeltraining kann Training in Gruppen Abwechslung und Motivation bringen.

Was kann man falsch machen?

- $\quad$ Pressen mit den Bauch-, Gesäss- oder Beinmuskeln blockiert Beckenbodenspannung

- $\quad$ Anhalten der Luft oder Pressen nach unten erhöhen den Druck im Bauchraum und verstärken die Inkontinenz

- Unterbrechen des Harnstrahls kann zur unvollständigen Entleerungen und zu Harnwegsinfekten führen

Was empfehlen wir zur Verbesserung der Versorgungslage der Patientinnen und Patienten mit COPD?

Wir empfehlen die Erfassung und die Behandlung von Harninkontinenz zukünftig in die medizinischen Routineuntersuchungen und Lungentrainingsprogramme einfliessen zu lassen.

Personen, die an COPD erkrankt sind empfehlen wir weiterhin:

- Vertrauen Sie sich Fachpersonen an. Sprechen Sie offen über Ihr Gesundheitsproblem.

- Achten Sie auf ausreichende Trinkmenge - etwa 2 Liter pro Tag.

- Führen Sie täglich Beckenbodenübungen durch, die Ihnen von spezialisierten Physiotherapeutinnen oder Physiotherapeuten gezeigt wurden.

- Aktivieren Sie Ihren Beckenboden vor und während des Hustens, sowie bei alltäglichen Aktivitäten, die zu Harnverlust führen.

- Informieren Sie sich über kompetente Angebote wie z.B. Empfehlungen des Deutschen Physiotherapie-Verbandes ZVK (www.physio-verband.de), der Deutschen Kontinenzgesellschaft DKG (www.kontinenz-gesellschaft.de) und Homepages spezialisierter Physiotherapeutinnen z.B. Pelvisuisse (www.pelvisuisse.ch).

Mein besonderer Dank gilt meinem Doktorvater, Herrn Prof. Dr. med. Dipl.-Ing. Helmut Teschler mit seinen Mitarbeitern, sowie Herrn Jens Lingemann und seiner Patientenorganisation Lungenemphysem-COPD Deutschland. Insbesondere danke ich allen Menschen, die freundlicher Weise an unserer Befragung teilgenommen haben.

\section{Barbara Köhler}

